SECTION IV

VARIABILITY 


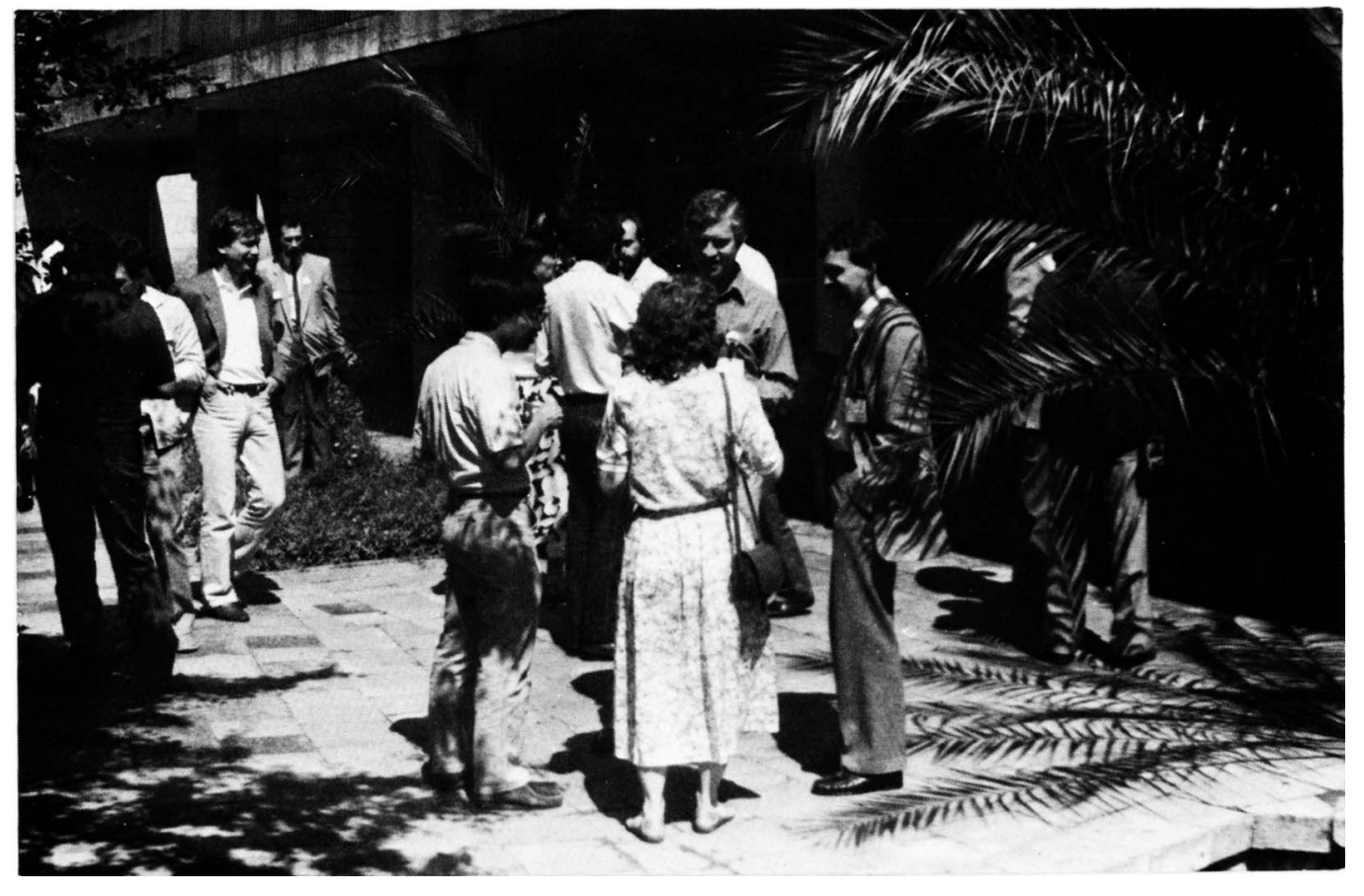

Peterson (right) with Sadler, Yee and Karachentsev during a break. 


\title{
SPECTRAL VARIABILITY IN SEYFERT GALAXIES
}

\author{
B.M. Peterson \\ Department of Astronomy \\ The Ohio State University \\ Columbus, Ohio 43210 \\ USA
}

\begin{abstract}
Recent observations of spectral variability in active galactic nuclei have established the connection between the broad emission-line and optical continuum flux changes. The inferred size of the broad-line region is at least an order of magnitude smaller than conventional estimates based on photoionization models, which leads to new conclusions about the nature of the broad-line region.
\end{abstract}

The variability of the optical and satellite ultraviolet spectra of active galaxies has been studied by a number of groups. Many of the important contributions in this area have been made by individuals in attendance at this symposium. The prinicpal result of studies to date is that the broad components of the permitted lines in Seyfert 1-type spectra vary with the continuum flux, with perhaps a short time delay between continuum and line variations. This was first recognized by Cherepashchuk and Lyutyi (1973) and has been reinforced by several subsequent studies. From this result, we can infer (1) that the broadline region (BLR) clouds are photoionized, (2) that the BLR clouds are optically thick in the Lyman continuum, and (3) that the BLR is considerably smaller than previously expected. While the first two conclusions are not surprising, the fact that the BLR is apparently very small requires us to rethink some of our assumptions about the BLR physics. The small size of the BLR has also, to a large extent, frustrated attempts to determine the BLR structure and kinematics by reverberation mapping (Blandford and McKee 1982; Capriotti, Foltz, and Peterson 1982).

Since the BLR is sufficiently small to be spatially unresolved in even the nearest active galaxies, the size of the BLR is conventionally determined on the basis of essentially thermodynamic (or, more precisely, ionization equilibrium) arguments. Energy input to the BLR depends on the 161

E. Ye. Khachikian et al. (eds.), Observational Evidence of Activity in Galaxies, 161-167.

(C) 1987 by the IAU. 
rate at which the central source produces ionizing photons,

$$
\mathrm{Q}(\mathrm{H})=\int_{\nu_{1}}^{\infty} \frac{\mathrm{L}_{\nu} \mathrm{d} \nu}{\mathrm{h} \nu}
$$

where $L_{\nu}$ is the specific luminosity of the source and the integral is over all energies in excess of one Rydberg. In addition to the shape of the ionizing spectrum and the chemical composition and column density of the BLR clouds, modern photoionization codes are characterized by an ionization parameter $U$, which is the ratio of ionizing photons to free electrons, i.e.,

$$
U=\frac{Q(H)}{4 \pi r^{2} \mathrm{cN}_{e}}
$$

Given fairly standard assumptions about the ionizing spectrum and chemical abundances, photoionization codes produce realistic relative line strengths for $U \simeq 10^{-2}$ and hydrogen column densities of $-10^{23} \mathrm{~cm}^{-2}$. The equation above can be solved for $r$, the separation between the BLR clouds and the continuum source, if $\mathrm{Q}(\mathrm{H})$ and $\mathrm{N}_{e}$ are known. The parameter $\mathrm{Q}(\mathrm{H})$ can be estimated by interpolating a power-law spectrum between satellite ultraviolet (i.e., near Ly $\alpha$ ) and X-ray (2-10 keV) measurements. The electron density is conventionally estimated from the absence of strong [O III] $\lambda \lambda 4363,4959,5007$, which requires densities higher than $-10^{8} \mathrm{~cm}^{-3}$, and the presence of C III] $\lambda 1909$, for which the critical density is $\sim 10^{10} \mathrm{~cm}^{-3}$. For two bright Seyfert 1 galaxies which we have studied extensively at Ohio State, Akn 120 and NGC 5548, we then estimate

$$
r \simeq \frac{1}{N_{9}} \text { light years }
$$

where $\mathrm{N}_{9}$ is the electron density in units of $10^{9} \mathrm{~cm}^{-3}$.

We can then ask on what time scales we expect to see variability in the broad emission lines. The time scale for variability of a small volume element of gas is determined by the recombination time $\tau_{R} \simeq 1 /\left(\mathrm{N}_{e} \alpha_{B}\right) \simeq$ $1 / \mathrm{N}_{9}$ hours. The time scale for response of a BLR cloud is similarly short since line photons diffuse in frequency and undergo a limited number of scatterings within the cloud. The response of the entire BLR is set by the light-travel time $\tau_{\mathrm{LT}}=\mathrm{r} / \mathrm{c} \simeq \mathrm{N}^{-1 / 2}$ years, as given above. It is worth noting at this point that for the narrow-line region, the recombination time $\left(-100 \mathrm{~N}_{3}^{-1}\right.$ years, where $\mathrm{N}_{3}$ is the electron density in units of $\left.10^{3} \mathrm{~cm}^{-3}\right)$ and the light-travel time $\left(-1000 \mathrm{~N}^{-1 / 2}\right.$ years $)$ are sufficiently long that narrow-line variability is not expected to be observable. 
On the basis of these estimates, we began monitoring a few variable Seyfert galaxies on a monthly basis in hopes of extracting information about the BLR kinematics from line-profile variability. However, our studies of Akn 120 (Peterson et al. 1983, 1985) and NGC 5548 (Peterson 1987) revealed that the broad $\mathrm{H} \beta$ fluxes in these galaxies change significantly in response to continuum changes on time scales much shorter than one year. Cross-correlation analyses of the Balmer-line and optical continuum fluxes in these galaxies yield response times of -5 days for Akn 120 and -35 days for NGC 5548 (Gaske11 and Sparke 1986; Peterson and Gaskell 1986); these time delays can be identified as the light-travel time between the continuum source and the region of peak broad-line emissivity. We therefore conclude that the BLR is at least 10 times smaller than predicted.

The inferred small size of the BLR implies that at least one of our initial assumptions is seriously in error. Possible explanations for the difference between the theoretical sizes and those inferred from variability include the following:

1. The ionizing continuum is at least 100 times weaker than estimated. This resolves the discrepancy by simply reducing $Q(H)$ and leaving the other parameters unchanged. Unfortunately the critical region of the spectrum cannot be observed directly in low-redshift objects. We can only note that there is no evidence for a significantly depressed Lyman continuum in high-redshift QSOs.

2. It might be supposed that the optical continuum changes lag behind changes in the ionizing continuum. This effectively increases $r$ without affecting the short response time between the optical continuum and $\mathrm{H} \beta$ variations. Again, it is difficult to test this hypothesis as we are unable to observe the critical region of the UV spectrum. However, examination of the $\mathrm{H} \beta$ variations alone implies that the BLR is still no larger than 1 - 2 light months, on the basis of coherence arguments.

3. It might be that the BLR electron density is indeed of order $10^{11} \mathrm{~cm}^{-3}$ or greater; this would allow a small BLR and a small ionization parameter, but it would imply that C III] $\lambda 1909$ is a poor indicator of $\mathrm{N}_{e}$ and probably also $U$. Part of the problem could be that some or even most of the emission ascribed to broad-line C III] $\lambda 1909$ may in fact have a different origin. One possibility is that narrow-line C III] $\lambda 1909$ may be a significant contributor (Ferland 1981); for the galaxies in question, the spectral resolution of IUE is insufficient to separate the narrow and broad components, but this should be relatively straightforward with Space Telescope. Another possibility is that some portion of the broad component may be attributable to species other than $\mathrm{C}^{++}$. For example, observations of the relatively narrow-line BAL QSO H0335-336 by Hartig and Baldwin (1986) reveal that the emission feature commonly identified as C III] $\lambda 1909$ is separated into three distinct components whose wavelengths agree well with the expected positions of three Fe III uv 34 lines. 
of these possible explanations, we favor the third. If indeed the region of peak emissivity in the BLR is characterized by clouds with $\mathrm{N}_{\mathrm{e}} \geqslant 10^{11}$ $\mathrm{cm}^{-3}$, it would seem that current photoionization models are missing the optimum domain in $\mathrm{N}_{e}$, $\mathrm{U}$ parameter space. Extension of photoionization models to higher densities should be a high priority.

The unexpectedly small size of the BLR has a number of interesting consequences. One of the most interesting is that the BLR $c$ an be virialized without requiring an extraordinary central mass. The mass required to gravitationally bind the BLR is

$$
M \simeq \frac{r v^{2}}{G} \simeq 5 \times 10^{6} r_{d} M_{\odot}
$$

where $r_{d}$ is the BLR radius in light days. This comfortably exceeds the the minimum mass required by the Eddington limit $\left(\sim 10^{6} \mathrm{M}_{\odot}\right)$, but it is not so large that it should be detectable in the rotation curve in the spatially resolved parts of the galaxies. It should be noted that the failure to detect extraordinarily massive central objects (i.e., 1010-11 $\mathrm{M}_{\mathcal{O}}$ ) from the rotation curves of nearby seyfert galaxies discredited virialized models - the discovery that the BLR is 10-100 times smaller than previously estimated revitalizes the idea that the BLR clouds are bound to the central source. The other major objection to virialized models has to do with cloud lifetimes, which are limited by Keplerian shear for rotation and by intercloud collisions for chaotic cloud motions. These problems are aggravated by a small BLR; however, the BLR crossing time for clouds is now so short that the lifetimes of radially moving clouds must also be very limited. There are apparently no simple kinematic models that ensure indefinite survival of individual BLR clouds.

If a massive collapsed object resides at the center of a small BLR, then radiation from the BLR is expected to be gravitationally redshifted (Netzer 1977). The expected magnitude of this redshift is

$$
\mathrm{c} \Delta \mathrm{z} \simeq \frac{\mathrm{GM}}{\mathrm{rc}} \simeq \frac{\mathrm{v}^{2}}{\mathrm{c}} \simeq 10^{2} \mathrm{~km} \mathrm{~s}^{-1}
$$

which is consistent with the observed small redward displacement of the Balmer lines relative to the forbidden lines in many active galaxies. While it is clear that other mechanisms are required to account for most of the wavelength displacements and profile asymmetries in active galaxy spectra, a small gravitational redshift is likely and must also be taken into account.

I am grateful to the National Science Foundation for their support of this work under grant AST83-17460 and to The Ohio State University for sponsoring my attendance at this symposium. 
References:

Blandford, R.F., and McKee, C.F. 1982, Astrophys. J., 255, 419.

Capriotti, E.R., Foltz, C.B., and Peterson, B.M. 1982, Astrophys. J., 261, 35.

Cherepashchuk, A.M., and Lyutyi, V.M. 1973, Astrophys, Lett., 13, 165.

Ferland, G.J. 1981, Astrophys, J., 249, 17.

Gaske11, C.M., and Sparke, L.S. 1986, Astrophys. J., 305, 175.

Hartig, G.F., and Baldwin, J.A. 1986, Astrophys. J., 302, 64.

Netzer, H. 1977, M.N.R.A.S., 181, 89P.

Peterson, B.M. 1987, Astrophys. J., 312, in press.

Peterson, B.M., Foltz, C.B., Miller, H.R., Wagner, R.M., Crenshaw, D.M., Meyers, K.A., and Byard, P.L. 1983, Astron. J., 88, 926.

Peterson, B.M., and Gaske11, C.M. 1986, Astron. J., 91, in press.

Peterson, B.M., Meyers, K.A., Capriotti, E.R., Foltz, C.B., Wilkes, B.J., and Miller, H.R. 1985, Astrophys. J,, 292, 164. 


\section{DISCUSSION}

TSVETANOV: What is the filling factor in the BLR?

PETERSON: The observations imply a smaller BLR than previously thoughi, but higher electron densities are then implied. Since the cloud emissivity increases as $\mathrm{Ne}^{2}$, a smaller BLR does not necessarily mean that the filling factor must be larger. In all cases studied, the filling factor is still less than $10^{-5}$ or thereabouts.

PREUSS: You said the narrow lines are basically constant. Can't you think of models which allow for variability? It would not be surprising if we hear during this symposium about observations reporting the variability oí narrow lines. (see next paper, eds.).

PETERSON: Narrow-line variability might be expected on timescales of several months, if the electron density is abnornally high or if the narrow-line region geometry is special. I am extremely suspicious of claims of short-term narrow-line variability since these measurements are very hard to make and many systematic errors can affect the data. The case for short-term variability should be examined closely (we should have learned a lesson from broad-line variability).

FILIPPENKO: Ground-based observations of high-redshift QSOs, as well as IUE observations of some Seyfert 1 galaxies having quite broad lines, provide support for your conclusion that CIII] comes from a different, lower-density region than permitted lines: CIII] $\lambda 1909$ often appears narrower than CIV $\lambda 1550$ and Ly $\alpha$ $\lambda 1216$.

PETERSON: I agree.

YEE: A few years ago, Oke and I looked at the variability of the BLRG's 3C 309.3 and 3C 382 (Yee \& Oke, Ap.J., 1982) over a ten-year period. We model the broad-line region with two components: a short-time response and long-time response (hence large size $\sim 1 \mathrm{pc}$ ) regions in order to explain the fact that the Balmer lines do not vary in direct proportionality to the variation of the continuum.

PETERSON: I wouldn't be at all surprised to find BLR gas as far away as 1pc. The cross-correlation technique we use gives only the separation between the continuur source and the radius of peak broad-line emissivity, not the spatial extent of the BLP.

TERLEVICH: Can you please comment on the total energy involved in these variations?

PETERSON: Over a few months, the luminosity in broad $H B$ can change by $\sim 10^{42}$ ergs $s^{-1}$.

MARASCHI: Do you have any incormation on the change of the shape of the continuum spectrum? 
PETERSON: After removing the stellar contribution in NGC 5548, the small portion of the optical spectrum that we observe ( $4000-6000 \AA)$ can be fitted with a power-law of index $0.5 \pm 0.1$ and no evidence for variability. This is consistent with results based on IUE spectra. The continuum shape in Akn 120 is being examined, but my initial impression is that it does not vary greatly.

HECKMAN: Have you looked at the relative time delay in the red and blue sides of the line profiles as a way of testing for infall or outflow in the BLR?

PETERSON: Yes, we have looked at the line profiles in NGC 5548 very carefully. During the 1984 observing season, the $\mathrm{HB}$ and $\mathrm{H} \gamma$ line profiles did not vary while the continuum and line fluxes increased. Since our observations span a period several times longer than the light-travel time across the BLR, our best guess is that most of the BLR is characterized by an isothermal distribution of cloud velocities. During the 1985 season, the profiles did not change, but they are different from the 1934 profiles in that a new component $\sim 2000 \mathrm{~km} \mathrm{~s}^{-1}$ blueward of line centre and around $2000 \mathrm{~km} \mathrm{~s}^{-1}$ wide has appeared (see also the paper by Chuvaev in this volume). From this observation, we conclude that some BLR gas comes from a physically distinct region perhaps one light-year from the nucleus (see also the comment by H.Yee). Profile analysis for Akn 120 is considerably more difficult on account of the extremely short response time of the BLR.

BOCHKAREV: 1) How many cases do you find with time delay between continuum and line variations? 2) Do you take account of variations of ionizing parameter inside BLR cloud?

PETERSON: 1) Based on our data and data from other published studies, a correlation between line and continuum variations has been establisined for around a half-dozen objects. The delay between continuum and line variations ranges from a few days to around a month. 2) Following the usual conventions, the ionization parameter we quote is appropriate for the inner face of a broad-line region cloud. The distance $r$ represents the distance of peak erissivity in the BLR. 